\title{
Health Informatics: Building Capacity Worldwide. Editorial
}

\author{
C. A. Kulikowskil, Antoine Geissbuhler ${ }^{2}$ \\ 'Department of Computer Science, Rutgers-The State University of New Jersey, New Brunswick, NJ, USA \\ ${ }^{2}$ Department of Imaging and Medical Informatics, Geneva University Hospitals, Geneva
}

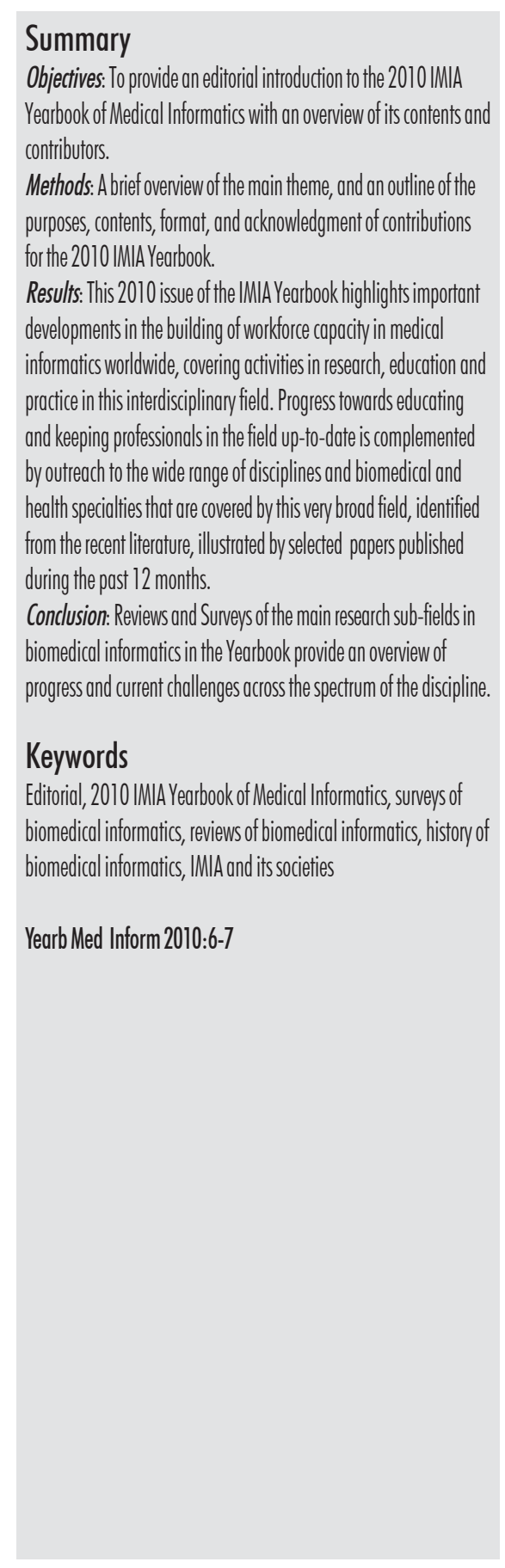

\section{Introducing the 2010 Yearbook and its Theme on Building Capacity Worldwide}

The Yearbook includes a number of articles focused on the theme of Health Informatics: Building Capacity Worldwide, covering present and past informatics contributions as well as future directions on how the field is addressing challenging problems of preparing professionals, researchers, and educators to deal with the increasing complexity of biomedical and health informatics in the $21^{\text {st }}$ Century. The motivation behind the theme is that we need to be educating, preparing and training more competent professionals across the globe in all areas covering the breadth of domains of our multi- and inter-disciplinary field. The essential role that biomedical informatics plays in the foundations of healthcare, follows from the increasing effectiveness and dissemination of informatics-enabled solutions. Today, considerable new challenges arise with attempts to scale-up activities in biomedical informatics both within countries and region. It is the role of IMIA to provide a global perspective that balances the needs of developed and developing regions of the world in bringing the latest advances in biomedical and health informatics to an increasingly wider community of interest. The purpose of this 2010 Yearbook of Medical Informatics to survey the progress achieved in our field over the past year, with its publication designed to coincide with the opening of the 2010 World Congress of Medical Informatics in Capetown, South Africa on September 13, emphasizing both the maturity and widespread impact of our discipline. The theme of Building Capacity Worldwide is especially relevant to help in achieving the high expectations of excellence in research in our field, that, combined with the diversity, breadth, and effectiveness of practical systems for healthcare, is what drives us forward internationally.

Major papers that comprise this 2010 IMIA Yearbook include:

Surveys:

- Chris Seegbrets (HF): Human Factors for Capacity Building

- Alvin Marcelo (HIS): Health Information Systems: Frameworks for Developing Countries

- Dan Rubin (SSII): Imaging Informatics: Toward Capturing and Processing Semantic Information in Radiology Images

- Georges Bediang (DS): Medical Decision Support Systems in Africa

- Chris Chute (KRM): Distributed Biomedical Terminology Development: From experiments to open process

- Heimar Marin (ECI): Nursing Informatics Education in the South: a Brazilian Experience

- Elke Lang (Bio): In Cereo and in Silico: Tissue Microarray (TMA) Techniques and Bioinformatics are Thriving Forces in Medical Science and Personalized Medicine

Reviews:

- Maurice Mars: Health Capacity Development Through Telemedicine in Africa

- Pradeep Ray: mHealth-an Ultimate Platform to Serve the Unserved

Research \& Education:

- Luo Shuqian, Zhang Kun and Li Baoluo (China): Medical Informatics in China: Healthcare IT 
trends, Academic and Research Developments

- Shashi Bhushan Gogia (India): An Overview of Education and Training of Medical Informatics in India

- Francis Roger France (Belgium): Board Certified Physicians in Health Informatics. A European Precedent for Professional Recognition

- Don Detmer: Capacity Building in eHealth and Health Informatics: A Review of the Global Vision and Informatics Educational Initiatives of the American Medical Informatics Association

History of MI: Randy Miller: A History of the INTERNIST-1 and Quick Medical Reference (QMR) ComputerAssisted Diagnosis Projects, with Lessons Learned.

\section{About the IMIA Yearbook of Medical Informatics}

The Yearbook of Medical Informatics of the International Medical Informatics Association (IMIA) is distributed through IMIA's Member and Corresponding Member Societies worldwide. Since its inception in 1992 it has been the single most important publication summarizing the activities of IMIA, and showcasing the best of medical informatics research for the previous year. Due to changing demands by its readership and the expanded availability of original papers over the web, the IMIA Yearbook of Medical Informatics has adopted a new format and mode of publication since 2006, with the goal of substantially broadening its availability to virtually all members of the IMIA family. In particular, surveys, giving overviews of recent developments, and comprehensive introductory reviews on relevant topics in the different fields of health and biomedical informatics, have been added as original articles. The Yearbook remains a non-profit publication of IMIA, jointly published with Schattauer Verlag. It is currently subscribed to by 23 of IMIA's member societies, providing access for about 20.000 individual members.

\section{Information on IMIA and on its Regional Groups}

The central challenge facing IMIA this year has been the preparations for its triennial conference MEDINFO, which will take place for the first time in Africa, in Cape Town in 2010.

The Yearbook contains detailed information about IMIA, its Member Societies, Regional Groups, Working Groups, and Special Interest Groups.

The section on IMIA Working Groups and Special Interest Groups was organized by Hyeoun-Ae Park. A detailed report on the activities of IMIA regions is included with the help of Regional Editors. We would like to thank Rolf Engelbrecht (for EFMI), Michio Kimura (for APAMI), Ghislain Kouematchoua (for HELINA), Alvaro Margolis (for IMIA LAC), Ramin Moghaddam (for MEAHI) and Karen Greenwood and RoseMary MacVicarElliot (for the North American IMIA Member Societies) for their valuable contributions.

\section{Acknowledgements}

The editors gratefully acknowledge the contributions of the referees and guest editors. They would also like to thank the authors of the invited surveys, reviews and the historical paper, and the contributors to the Research and Education Section. They are most appreciative of the considerable skill, time, and effort devoted by Christian Lovis, the Managing Editor, and by the Section Editors, Claudine Bréant, Célia Boyer, Adrien Depeursinge, Arnaud Gaudinat, Alberto Guardia, Daniel Kubias, Rodolphe Meyer, Henning Müller, Anne-Marie Rassinoux, Patrick Ruch, and Lina Yip. They especially wish to thank the Editorial Assistant, Martina Hutter, from the Medical Faculty at the University of Heidelberg, without whose untiring efforts the Yearbook would not have been completed. The editors appreciate the contributions of the Advisory Board to the planning of this Yearbook. They are Reinhold
Haux, Fernando Martin-Sanchez, Georges Mihalas, and Peter Murray.

The referees who contributed to the selection of articles in the 2010 Yearbook of Medical Informatics were:

Jos Aarts, The Netherlands

Ameen Abu-Hanna, The Netherlands

Kouhei Akazawa, Japan

Sameer Antani, USA

Fernan Gonzalez Bernaldo de Quiros, Argentina

Olivier Bodenreider, USA

Alex A.T. Bui, USA

Yu Cao, USA

Karen Courtney, USA

Dina Demner Fushman, USA

Georg Duftschmid, Austria

Martin Dugas, Germany

John Glaser, USA

Natalia Grabar, France

Stefan Gräber, Germany

Ghassan Hamarneh, Canada

Heinz Handels, Germany

Dimitar Hristovski, Slovenia

Katharina Kaiser, Austria

Theodore Kalamboukis, Greece

Jayashree, Kalpathy-Cramer, USA

Jeongeun Kim, Korea

Pablo Laguna, Spain

Andy H. Lee, Australia

Luigi Lo Iacono, Germany

Daniel Luna, Argentina

Yasuhsi Matsumura, Japan

Stéphane Meystre, USA

Anne Moen, Norway

Maryati Mohd Yusof, Malaysia

Jeannette Murphy, UK

Toshiharu Nakai, Japan

Michael Poprat, Germany

Daniel Racoceanu, Singapore

Dietrich Rebholz-Schuhmann, UK

Rainer Röhrig, Germany

Indra Neil Sarkar, USA

Michael Schumacher, Spain

Arash Shaban-Nejad, Canada

Guy Tsafnat, Australia

Manolis Tsiknakis, Greece

Frank Ückert, Germany

Devrim Ünay, Turkey

Mauno Vihinen, Finland

Ulrike Wittig, Germany

Toru Yao, Japan

Tatjana Zrimec, Australia 